\title{
PREDICTION OF CLINICAL SYMPTOMS OF SCHIZOPHRENIA BASED ON COMT METHYLATION MARKER
}

Nour El Huda Abd Rahim ${ }^{1}$, Mohd Nabil Fikri Rahim ${ }^{1}$, Norsidah Ku Zaifah ${ }^{1}$, Hanisah Mohd Noor $^{2}$, Kartini Abdullah ${ }^{2}$ and Norlelawati A. Talib ${ }^{3}$

${ }^{1}$ Department of Basic Medical Sciences, Kulliyyah of Medicine, International Islamic University Malaysia, Pahang, Malaysia, ${ }^{2}$ Department of Psychiatry, Kulliyyah of Medicine, International Islamic University Malaysia, Pahang, Malaysia, ${ }^{3}$ Department of Pathology and Laboratory Medicine, Kulliyyah of Medicine, International Islamic University Malaysia, Pahang, Malaysia.

Presenter: Nour El Huda binti Abd Rahim, elhuda@iium.edu.my

Introduction: The dopamine hypothesis of schizophrenia is based on the fact that hyperdopaminergic state is involved in causing psychosis and antipsychotic drugs block the dopamine receptor. COMT regulates the homeostatic levels of neurotransmitter dopamine in the synapses and plays a role in the neurocognitive function. The dysregulation of dopamine in the prefrontal cortex influences the cognitive function and the severity of the psychotic symptoms in schizophrenia. During epigenetic event, methylated COMT gene may cause reduction in its expression and contribute to the clinical presentation of schizophrenia. Therefore, the aim of this study was to assess the feasibility of using COMT DNA methylation for the prediction of specific psychotic presentation of schizophrenia.

Materials and method: In this study, 138 schizophrenia patients were recruited from the Psychiatry Clinic, Hospital Tengku Ampuan Afzan, Kuantan Pahang. Genomic DNA from peripheral blood was subjected to the Methylight Taqman ${ }^{\circledR}$ analysis for quantitative measurement of the COMT DNA methylation. The psychopathological symptoms were assessed using the Positive and Negative Syndrome Scale (PANSS).

Results: The regression analysis showed that the Positive and Excited subdomains of PANSS were significant predictors of COMT hypomethylation $(B=-0.288, p=-0.031 ; B=-$ $0.288, p=-0.031)$. The Excited subdomain of PANSS was negatively correlated with COMT DNA methylation $\left(r^{2}=-0.380, p=0.000\right)$ as well as the Depressed subdomain $\left(r^{2}=-0.288\right.$, $p=0.001)$.

Conclusion: The relationship between DNA methylation of COMT with the positive, excited and depressed symptoms might indicate the epigenetic role of COMT gene in the manifestation of schizophrenia. 